\title{
A commutativity theorem for rings
}

\section{Steve Ligh and Anthony Richoux}

Let $R$ be a ring with an identity and for each $x, y$ in $R$, $(x y)^{k}=x^{k} y^{k}$ for three consecutive positive integers $k$. It is shown in this note that $R$ is a commutative ring.

It is well known that each of the following conditions on any group $G$ insures that $G$ is commutative:

(i) for each $x, y$ in $G,(x y)^{2}=x^{2} y^{2}$;

(ii) for each $x, y$ in $G,(x y)^{k}=x^{k} y^{k}$ for three consecutive positive integers $k$.

Several authors have considered the ring-theoretic analogues of the above group-theoretic results $[1,2,3,4,5,6,7]$. Johnsen, Outcalt and Yaqub have shown in [5] that if $R$ is any nonassociative ring with 1 such that $(x y)^{2}=x^{2} y^{2}$ for all $x, y$ in $R$, then $R$ is commutative. Furthermore, they provided examples showing that for any integer $k>2$, there exists a noncommutative ring $R$ with $I$ satisfying the identity $(x y)^{k}=x^{k} y^{k}$ for all $x, y$ in $R$. For the ring-theoretic analogue of (ii), a partial solution was given by Luh [6]. He showed that any primary ring having the condition $(x y)^{k}=x^{k} y^{k}$ for three consecutive positive integers $k$ is commutative. The purpose of this note is to furnish a complete, but elementary, solution of the ring-theoretic analogue of (ii).

THEOREM. If $R$ is a ring with 1 which satisfies the identities $(x y)^{k}=x^{k} y^{k}, k=n, n+1, n+2$, where $n$ is a positive integer, then $R$ is commutative.

Received 26 August 1976. 
Proof. Let $x, y$ be in $R$. From $x^{n+1} y^{n+1}=x^{n} y^{n} x y$, it follows that

$$
x^{n}\left(x y^{n}-y^{n} x\right) y=0
$$

Since (1) holds for all $x, y$ in $R$, substitute $(x+1)$ for $x$ and simplify, to get

$$
(x+1)^{n}\left(x y^{n}-y^{n} x\right) y=0
$$

Multiply (2) on the left by $x^{n-1}$ and expand $(x+1)^{n}$ by the binomial theorem, keeping in mind the identity (1); it follows that

$$
x^{n-1}\left(x y^{n}-y^{n} x\right) y=0 \text {. }
$$

Since (3) is valid for each $x, y$ in $R$, continue the above process, that is, replace $x$ by $(x+1)$ and multiply (3) on the left by $x^{n-2}$ eventually one gets

$$
x\left(x y^{n}-y^{n} x\right) y=0
$$

Again substitute $(x+1)$ for $x$ and use (4), to get

$$
\left(x y^{n}-y^{n} x\right) y=0
$$

Now from the identity $x^{n+2} y^{n+2}=x^{n+1} y^{n+1} x y$, we have

$$
x^{n+1}\left(x y^{n+1}-y^{n+1} x\right) y=0 \text {. }
$$

Enploying the same technique used to get (5) from (1), one obtains

$$
\left(x y^{n+1}-y^{n+1} x\right) y=0 \text {. }
$$

Multiply both sides of (5) on the left by $y$, to get

$$
y x y^{n+1}=y^{n+1} x y \text {. }
$$

From (7) and (8) we have

$$
(x y-y x) y^{n+1}=0 .
$$

Now apply the same technique used to get (5) from (1), this time substituting $(y+1)$ for $y$; we then have

$$
(x y-y x) y=0
$$


Finally replace $y$ by $(y+1)$ and use (10), to obtain $x y-y x=0$. Thus $R$ is commutative.

\section{References}

[1] Howard E. Bell, "On a commutativity theorem of Herstein", Arch. Math. (Basel) 21 (1970), 265-267.

[2] Howard E. Bell, "On some commutativity theorems of Herstein", Arch. Math. (Basel) 24 (1973), 34-38.

[3] A.H. Boers, "A note on a theorem on commutativity of rings", $K$. Nederl. Akad. Wetensch. Proc. Ser. A 72 = Indag. Math. 31 (1969), 121-122.

[4] I.N. Herstein, "Power maps in ringss", Michigan Math. J. 8 (1961), 29-32.

[5] E.C. Johnsen, D.L. Outcalt and Adil Yaqub, "An elementary commutativity theorem for rings", Amer. Math. Monthly 75 (1968), 288-289.

[6] J. Luh, "A commutativity theorem for primary rings", Acta Math. Acad. Sci. Hungar. 22 (1971), 211-213.

[7] Walter Streb, "Über die Potenzgesetze", Enseignement Math. (2) 20 (1974), 223-225.

\footnotetext{
Department of Mathematics,

University of Southwestern Louisiana,

Lafayette,

Lou is i ana,

USA.
} 